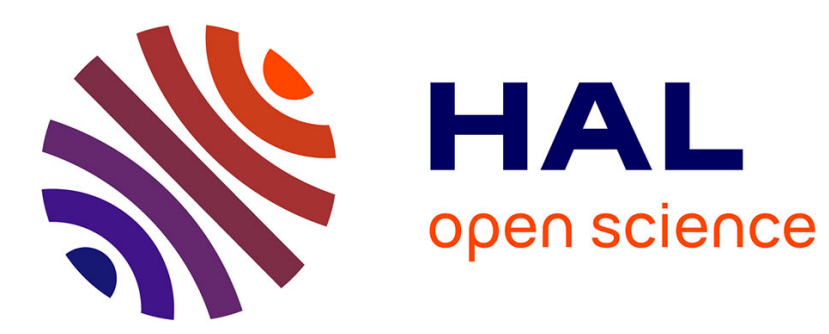

\title{
Appropriation de nouvelles ressources d'enseignement par des professeurs de physique-chimie en seconde
}

Laurent Jeannin, Laurent Veillard, Andrée Tiberghien

\section{To cite this version:}

Laurent Jeannin, Laurent Veillard, Andrée Tiberghien. Appropriation de nouvelles ressources d'enseignement par des professeurs de physique-chimie en seconde. RDST - Recherches en didactique des sciences et des technologies , 2010, 1, pp.267-292. hal-00965209

\section{HAL Id: hal-00965209 \\ https://hal.science/hal-00965209}

Submitted on 24 Mar 2014

HAL is a multi-disciplinary open access archive for the deposit and dissemination of scientific research documents, whether they are published or not. The documents may come from teaching and research institutions in France or abroad, or from public or private research centers.
L'archive ouverte pluridisciplinaire HAL, est destinée au dépôt et à la diffusion de documents scientifiques de niveau recherche, publiés ou non, émanant des établissements d'enseignement et de recherche français ou étrangers, des laboratoires publics ou privés. 


\section{Appropriation de nouvelles ressources d'enseignement par des professeurs de physique chimie en seconde: cas d'une séquence en mécanique}

Titre en Anglais: Appropriating new tools for teaching: the case of physics teachers at upper secondary school in France.

Auteurs : Laurent Jeannin, Laurent Veillard et Andrée Tiberghien

UMR ICAR 5191 (CNRS, Université Lumière Lyon 2, ENS, INRP), équipe COAST

15 Parvis René Descartes BP 700069342 Lyon Cedex 07 FRANCE

Contacts : laurent.jeannin@univ-lyon2.fr, laurent.veillard@univ-lyon2.fr,

andree.tiberghien@univ-lyon2.fr

\section{Préversion d'un article paru dans la revue $\operatorname{RDST}^{2010,} \mathbf{n}^{\circ} 1$, pp. 267-292}

Résumé. Cet article traite de l'appropriation de nouvelles ressources pour l'enseignement d'une séquence sur la mécanique par des professeurs de physique-chimie en seconde. Il s'agit de ressources conçues par des groupes de chercheurs et d'enseignants, dans le cadre d'une démarche de recherche - développement engagée depuis plusieurs années. Nous proposons un cadre théorique issu de la théorie de l'activité instrumentée de Leontiev et d'approches en didactique des mathématiques pour analyser ce processus d'appropriation. La méthodologie développée s'appuie largement sur l'analyse d'enregistrements vidéo de : 1) l'activité de trois enseignants qui se retrouvent régulièrement entre les séances d'enseignement pour s'approprier ces ressources et discuter de leurs choix de mise en ouvre dans leur classe ; 2) l'activité de l'un de ces enseignants dans sa classe, avec ses élèves. Les résultats montrent que les enseignants reconnaissent la faisabilité dans la classe de ce que ces ressources proposent, cependant ils pointent une difficulté des enseignants à saisir les choix épistémologiques des concepteurs.

Mots clés : Ressources d'enseignement ; mécanique ; seconde ; activité de l'enseignant ; apprentissage des élèves, analyse vidéo

Abstract. This article deals with the question of the appropriation by physics-chemistry teachers of new resources for the teaching of a sequence on the mechanics in secondary school. The resources were built by groups of researchers and teachers within the framework of an approach of research and development engaged for several years. We propose a theoretical framework based from the theory of the activity instrumented by Leontiev and by approaches in didactics of the mathematics to analyze this process of appropriation. The methodology is widely founded on the video recordings analysis of: 1) the activity of three teachers who meet regularly between the sessions of teaching to appropriate these resources and discuss their choices of implementation in their class; 2) the activity of one of these teachers in his class, with his student. The results show that teachers recognize the feasibility of the implementation of these resources in a class, however they show teachers'difficulty of understanding the epistemological choices of the designers.

Keywords: resources for teaching; mechanics; secondary school; teacher's activity; students' learning ; video analysis 


\section{Introduction}

Depuis plusieurs années, des chercheurs en didactique de la physique du laboratoire ICAR (Interaction Corpus, Apprentissage, Représentation, http://icar.univ-lyon2.fr/) et des enseignants du secondaire collaborent à la création et à la diffusion d'outils et de ressources pour l'enseignement de cette discipline. Il s'agit notamment de proposer des séquences d'enseignement conformes aux programmes nationaux, accompagnées d'activités, de vidéos d'élèves montrant certaines difficultés de compréhension et de les mettre à disposition du plus grand nombre d'enseignants via un site internet ou un support DVD. Cependant, la question se pose de la manière dont ces derniers s'approprient effectivement ces ressources. Des recherches se développent depuis quelques années pour mieux comprendre les ressources mobilisées par les enseignants et, dans certains cas, pour évaluer les effets de nouvelles ressources proposées (notamment numériques) sur l'enseignement effectif dans les classes (Gueudet \& Trouche, 2009, 2010; Lagrange, Lecas , \& Parsysz, 2006). Cependant, il existe encore peu de travaux de ce type dans le domaine des sciences expérimentales, qui s'intéressent notamment à l'appropriation de nouvelles ressources par des enseignants et leur effet sur l'activité de la classe.

Cet article, réalisé à partir d'un travail de thèse (Jeannin, 2006) veut contribuer à la problématique de l'appropriation de nouvelles ressources par un enseignant dans le champ de l'enseignement de la physique. Notre premier objectif est de proposer un cadre théorique et une méthodologie associée, pour traiter de ce type de questions. Puis, à partir de l'observation de réunions regroupant des enseignants de physique-chimie en lycée (seconde) discutant de la mise en œuvre possible d'une nouvelle séquence en mécanique, et du suivi d'un de ces enseignants dans sa classe, nous essayerons de construire un certain nombre d'hypothèses quant aux procédures et questions posées par cette appropriation.

L'article s'articule en trois parties. En premier lieu, nous présentons la séquence construite par le groupe de chercheurs et d'enseignants, notamment les choix épistémologiques sous-jacents. Puis nous explicitons le cadre théorique et la méthodologie d'analyse des données sur lesquels nous nous sommes appuyés pour étudier l'appropriation de cette séquence par un enseignant dans sa classe. Enfin, nous présentons les résultats de l'étude de l'appropriation de cette séquence par un groupe d'enseignant dans une phase préparatoire, et sa mise en œuvre dans une classe par un de ces enseignants.

\section{Présentation de la séquence conçue par le groupe de $R \& D$}

La séquence a été conçue par un groupe de recherche - développement composé d'enseignants et de chercheurs, appelé groupe Sésames (Situations d'Enseignement Scientifique : Activités de Modélisation, d'Évaluation, de Simulation). Une caractéristique de ce groupe est d'avoir élaboré des outils théoriques de conception de séquences (Tiberghien, Vince, \& Gaidioz, 2009) fondés sur une analyse épistémologique de la modélisation en physique et sur les hypothèses d'apprentissage liées. La séquence propose un ensemble théorique explicite qui permet d'interpréter diverses situations et de prévoir quelques faits expérimentaux. Cette activité de conception a permis de proposer des séquences d'enseignement composées d'activités pour les élèves et de commentaires sur cinq points : le but de l'activité, sa préparation, le savoir en jeu, le comportement des élèves et la correction. Ces séquences proposent une forme d'enseignement qui, au lycée, se détache de la traditionnelle distinction entre cours et $T P$. Le nouveau savoir est introduit par des activités réalisées par les élèves. Lors de la mise en commun, les élèves font des propositions qui sont débattues. Le professeur procède ensuite à une institutionnalisation. Dans certains cas le nouveau savoir est introduit en début d'activité sous la forme d'un texte. Les activités visent alors à aider les élèves à comprendre et mettre en œuvre les concepts introduits. 
La mise en œuvre de certaines des séquences, dont celle qui fait l'objet de ce travail, ont été étudiées par des chercheurs. Ces chercheurs ont filmé une série de séances en classe (Coulaud, 2005 ; Küçüközer, 2005). L'analyse des vidéos a permis de sélectionner des extraits qui illustrent certains comportements d'élèves. Ainsi la rubrique «comportements des élèves » présente dans certains cas des extraits vidéo correspondant à l'activité. Au moment où les données ont été récoltées pour cette étude, un site était en cours d'élaboration, et les professeurs ont pu utiliser pour certaines réunions un $\mathrm{CD}$ qui préfigurait ce site. Ce site est appelé PEGASE (pegase.inrp.fr).

\section{Approche théorique de l'activité enseignante}

\section{Quelques aspects importants de l'activité enseignante issus des recherches}

Initiées aux Etats-Unis dès les années 50, les études sur la pratique, l'action ou l'activité enseignante (les termes diffèrent selon les orientations théoriques) ont donné lieu à de nombreuses publications (Bressoux, et al., 2002; Brophy, 1983; Crahay, 1989; Doyle, 1983, 1986; Walberg \& Fowler, 1991). D'abord centrés sur la recherche de critères du bon enseignant, puis celle de corrélations entre processus et produits, d'autres approches théoriques ont été ensuite développées, multipliant ainsi les points de vue sur cet objet d'étude complexe : approches cognitivistes (Shavelson \& Stern, 1981; Tochon, 1993), écologiques (Bronfenbrenner, 1986), de la cognition située (Durand, 1996; Gal-Petitfaux \& Saury, 2002), ergonomiques (Amigues \& Faïta, 2001; Rogalski, 2000) ou encore interactionnistes et plurielles (Altet \& Vinatier, 2008; Robert \& Rogalski, 2005).

Nous retenons de cette somme considérable de travaux, quelques points clés pour caractériser l'activité enseignante dans notre étude.

En premier lieu, celle-ci ne se réduit pas au face à face de l'enseignant avec les élèves dans la classe, mais comporte toute une partie réalisée hors de la classe. Robert (1999) suggère, par exemple, de distinguer « pratiques enseignantes » qui se déroulent hors de la classe et " pratiques en classe ». Ces deux aspects sont liés, l'un impactant l'autre, mais en même temps peuvent être dotés d'une relative indépendance.

Les études portant sur les planifications des enseignants, c'est-à-dire sur la manière dont ceux-ci préparent, hors de la classe, les séances en classe, montrent que ceux-ci sont généralement préoccupés par trois principaux éléments de la situation d'enseignement : le contenu enseigné, qui est l'élément le plus prégnant, suivi des caractéristiques des élèves et de manière moindre, du matériel disponible (Shavelson \& Stern, 1981).

Les recherches portant sur les liens entre ces planifications et l'activité en présence des élèves, curieusement peu nombreuses alors que les études centrées sur l'un ou l'autre de ces deux moments de l'activité de l'enseignant sont assez fournies, mettent en évidence des écarts parfois importants entre anticipations et réalisations effectives en classe (Durand, 1996). Ainsi, Bru (1991) montre qu'il existe une grande variabilité entre les enseignants sur le plan de la planification: tous ne sont pas autant planificateurs et des décisions in situ peuvent conduire à des abandons de certaines prévisions. Altet (1994) va également dans ce sens et met en évidence que les décalages sont même souvent nécessaires pour pouvoir prendre en compte des évènements non prévisibles, comme par exemple des difficultés de compréhension des élèves ou des aléas matériels. Les recherches menées depuis quelques années dans le paradigme de la cognition située insistent sur ce dernier point, en considérant le fait que les planifications sont, au mieux, une ressource parmi d'autres pour l'activité en classe et qu'elles servent surtout à établir et peaufiner des routines d'enseignement (Durand, 
1996; Leinhardt \& Greeno, 1986) qui seront utilisées de manière située, en fonction des évènements.

Nous retenons ici l'idée qu'il est crucial, dans la perspective d'une problématique centrée sur l'appropriation d'une nouvelle séquence d'enseignement par un enseignant, de s'intéresser non seulement à son activité préparatoire et planificatrice, ou à l'inverse, seulement aux réalisations en classe, mais également de se pencher sur ce qui se passe effectivement en classe, et de s'intéresser à la mise en lien entre ces deux moments, avec la question des instruments ou artefacts permettant cette mise en lien.

Un autre point important ressort de travaux menés en ergonomie (voir Amigues, 2002 pour une revue des travaux) ou en didactique professionnelle (Rogalski, 2000). Ces travaux montrent que l'expertise enseignante se construit dans l'activité située dans des contextes matériels et symboliques spécifiques, mais aussi en réponse à des prescriptions institutionnelles. L'expertise se traduit notamment par la constitution progressive d'invariants dans l'activité : la notion d'organisateurs de l'activité enseignante, au cœur des travaux du réseau OPEN ${ }^{1}$ par exemple indique très clairement l'importance de cette idée d'invariance que l'on retrouve aussi dans beaucoup d'autres travaux. Ces invariants sont pour une part propres à un individu donné, et pour une part, plus largement partagés par un collectif professionnel, ce qu'expriment notamment les notions de genre et de style (Clot, 1999) par exemple.

Enfin, des travaux plus récents sur l'utilisation de ressources par les enseignants en didactique des mathématiques, mettent en évidence que les systèmes de ressources (programme, manuels scolaires, sites internet, etc.) avec lesquels ils interagissent constituent une caractéristique structurée et structurante de leur pratique hors classe et en classe (Gueudet \& Trouche, 2010 ; Lahire, 1998). Nous retenons de ces travaux que les professeurs entretiennent une relation de nature dialectique avec ces ressources : d'une part ces dernières, selon leurs caractéristiques (tant sur le plan du contenu épistémologique que de la mise en forme discursive) contribuent significativement à façonner l'activité du professeur dans un mouvement d'instrumentation ; d'autre part les connaissances du professeur, ses modes d'engagement, ses intentions et ses décisions didactiques, orientent sa documentation, dans un mouvement d'instrumentalisation.

\section{Un acteur pluriel}

Dans cette étude, nous proposons de considérer l'enseignant comme un acteur pluriel, pour reprendre un terme proposé par Lahire (1998). Nous signifions par là qu'un enseignant n'est pas réductible à son activité située ou à ses routines d'enseignement dans la classe, pas plus qu'à ses planifications, anticipations ou retours réflexifs hors de la classe, ou encore aux prescriptions institutionnelles qu'il reçoit. Ce sont là différentes composantes de son activité qui en font la complexité et qui sont plus ou moins liées. Notre hypothèse est qu'il est important de les prendre en compte sans les isoler les unes des autres, dans la perspective d'une étude sur l'appropriation d'une nouvelle séquence d'enseignement.

Dans cette optique, la théorie de l'activité proposée par Leontiev (1979), comme modèle d'activité médiatisée par des instruments et articulant 3 niveaux (Activité, action, opération) nous semble une approche intéressante pour conserver ces différents aspects.

(10) Les actions de l'enseignant s'inscrivent dans des activités collectives qui répondent elles-mêmes à une certaine motivation ou finalité sociale (ex : préparer une séquence d'enseignement en physique; enseigner cette séquence dans une classe, etc.) et à des 
prescriptions institutionnelles plus précises ; ces activités peuvent s'étendre sur des périodes de temps parfois relativement importantes.

(10) Les actions de l'enseignant sont (définies au travers du flux continu de l'activité, elles s'étalent sur une plus courte durée pas très clair); elles sont médiatisées par des instruments matériels et symboliques et sont orientées par des buts ou intentions conscientes. C'est l'enseignant qui produit et guide ses actions.

(10) Les opérations sont des routines ou des singularités définies en réponse à des conditions concrètes rencontrées lors de l'exécution d'une action que l'enseignant sollicite individuellement de manière consciente ou inconsciente.

Dans cette approche, une séquence d'enseignement et les ressources proposées par le groupe Sésames à l'enseignant pour la mise en œuvre dans sa classe sont considérées comme un ensemble d'instruments, dotés d'une cohérence épistémologique et d'une mise en forme discursive propres dont l'enseignant n'a pas connaissance a priori. Il s'agit de savoir comment ces nouveaux instruments vont être compris et pouvoir s'insérer dans la pratique habituelle de l'enseignant.

\section{Spécification du modèle générale d'activité à l'enseignement}

Le modèle général de Leontiev ne nous semble pas suffisamment précis pour constituer un cadre théorique de l'activité enseignante. Pour le compléter, nous reprenons tout d'abord la distinction opérée par Robert (1999) entre «activité enseignante », dans la classe et «activité hors classe » (cette dernière étant pour nous principalement abordée dans la préparation des séances de classe). Nous postulons qu'il y a des liens entre ces deux moments de l'activité enseignante, mais qu'ils se déploient dans des temporalités et des environnements spécifiques, générateurs de contraintes assez différentes et qui tendent à leur donner une relative indépendance.

\section{L'activité préparatoire hors classe}

$\mathrm{Au}$ cours de la préparation de ses séances de classe, l'enseignant que nous avons observé se trouvait dans deux types de situations.

A certains moments, il était avec deux autres enseignants du même établissement lors de réunions de préparation collective dont l'objectif était de s'approprier la nouvelle séquence proposée et de réfléchir à sa mise en œuvre. Ces réunions se déroulaient dans l'enceinte de l'établissement (salle des professeurs de physique). Nous proposons de considérer ces réunions comme un moment d'activité collective, dont les actions seront principalement réalisées par des opérations de nature communicative. Dans ce premier type d'activité, la notion de but, liée au niveau de l'action dans le modèle de Leontiev, mérite qu'on la discute car elle renvoie à l'idée d'une intention claire de la part de l'enseignant qui va prendre la parole. Nous proposons de parler plutôt ici de préoccupation sur des objets donnés, qui conserve l'idée d'une intention consciente, mais parfois assez imprécise quant au but précis à atteindre. Une préoccupation n'est pas nécessairement déclenchée en réunion. Elle peut naître lors de la préparation personnelle d'un intervenant ou lors de l'enseignement proprement dit.

Finalement nous caractérisons une action lors de ces réunions par :

- un objet ou plusieurs objets de préoccupation (ex : un contenu d'enseignement ; la réaction des élèves ; etc.) qui l'initie(nt) et l'oriente(nt) ;

- des ressources auxquelles fait appel le locuteur/acteur pour contribuer à la discussion (ex : ses connaissances personnelles, les documents proposés dans la séquence ; etc.) 
- sa nature cognitive (ex : analyser un contenu d'enseignement ou comparer le savoir en jeu avec les difficultés prévisibles des élèves).

Nous détaillons les différents types d'objets, de ressources et de nature cognitive dans la partie méthodologique.

\section{L'activité enseignante en classe}

L'activité dans la classe est appréhendée ici comme une activité collective, mettant en jeu l'enseignant et ses élèves et médiatisée par des instruments. Cette activité est liée à l'activité préparatoire, grâce à des artefacts ou instruments permettant la mise en lien. La préparation de l'enseignant constitue un instrument privilégié dans cette perspective: elle donne à l'enseignant des «lignes d'actions » (Robert, 1999) qu'il pourra plus ou moins mettre en œuvre selon les circonstances.

Dans la classe, les actions de l'enseignant sont orientées par des buts et sont mises en œuvre par des opérations, là encore principalement de nature communicative (discours oraux et écrits, gestes signifiants, etc.).

Les notions de contrat didactique et de milieu définies en didactique des mathématiques (Brousseau, 1998) sont ici intéressantes pour préciser ce modèle général. Elles permettent de disposer d'outils théoriques pour décrire l'évolution dans le temps :

- d'une part les règles du jeu entre enseignant et élèves par rapport au savoir en jeu dans la séance et plus précisément ce que chacun est censé faire ou de ne pas faire ;

- des objets, matériels et symboliques, qui constituent le contexte de l'activité de l'enseignant et de ses élèves.

A partir de cette approche théorique, notre recherche a pour objectif de caractériser les modes d'appropriation des ressources proposées et leur impact sur l'activité enseignante, en analysant plus précisément :

- ce qui préoccupe les enseignants au départ puis au cours des différentes réunions préparatoires et oriente leurs actions préparatoires des séances ;

- les liens entre a) ce qui se passe lors de ces séances d'échanges et b) les choix d'un enseignant, ses actions réalisées dans la classe et leurs effets sur l'activité des élèves.

\section{Méthodologie}

Nous avons fait le choix de suivre plus particulièrement un enseignant (A), dans une approche longitudinale sur un temps relativement long ( séquence d'enseignement s'étalant sur plusieurs mois). Ce choix est cohérent avec nos options théoriques et notre problématique dans la mesure où il ne nous semble pas possible de véritablement étudier l'appropriation d'une séquence sur un temps court.

\section{Recueil des données}

Comme on a pu déjà l'évoquer dans la partie théorique, nous avons pu observer et enregistrer A dans 2 types de situations :

1) des réunions de préparation où il échangeait avec deux de ses collègues également professeurs de physique chimie dans ce lycée, à propos de cette séquence. Nous souhaitions analyser l'activité préparatoire des séances, au moment où l'enseignant prend connaissance de la séquence et des outils proposés. Cette phase de l'activité enseignante est difficile d'accès : elle se passe chez lui, à des moments difficiles à prévoir, et donne lieu à peu de verbalisations. Nous avons donc profité de ce dispositif, réunissant cet enseignant avec deux autres de ses collègues. Il a le grand avantage d'être très favorable à une observation et un enregistrement 
vidéo. Chaque réunion de préparation a été filmée avec une caméra en grand angle placée dans un coin de la salle (Figure 1); Nous avons effectué des zooms pour conserver la trace de l'utilisation des documents Outils tout en sachant que nous perdions, dans le même temps, des informations sur le comportement des enseignants ;

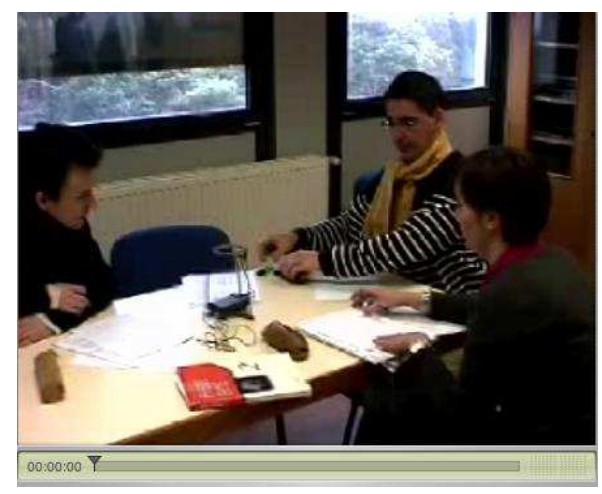

Figure 1 : cadrage des vidéo des préparations

2) les séances d'enseignement en classe avec les élèves. Lors de ces séances de classe, nous avons filmé simultanément l'enseignant et deux binômes d'élèves. Les binômes étaient filmés à l'aide d'une caméra fixe dont le champ comprenait la paillasse et les élèves avec un microcravate pour chacun. L'enseignant était filmé en utilisant une caméra et un micro sans fil HF selon le point de vue de «l'élève idéal » (Stigler, Gonzales, et al., 1999).

Notre corpus de données est donc constitué :

- des enregistrements vidéo des réunions (6 fois $45 \mathrm{~min}$ );

- des enregistrements vidéo des séances d'enseignement : une vidéo centrée sur l'enseignant du point de vue de «l'élève idéal » et deux centrées sur des binômes d'élèves (24h pour 1 'enseignant et 2 fois $24 \mathrm{~h}$ pour les élèves) ;

- des documents personnels de l'enseignant rédigés pour chaque séance (12 documents) ;

- des documents distribués aux élèves au cours des séances en classe.

La figure 2 donne la répartition dans le temps de la succession des réunions et des séances.

Figure 2 : répartition des séances et réunions enregistrées)

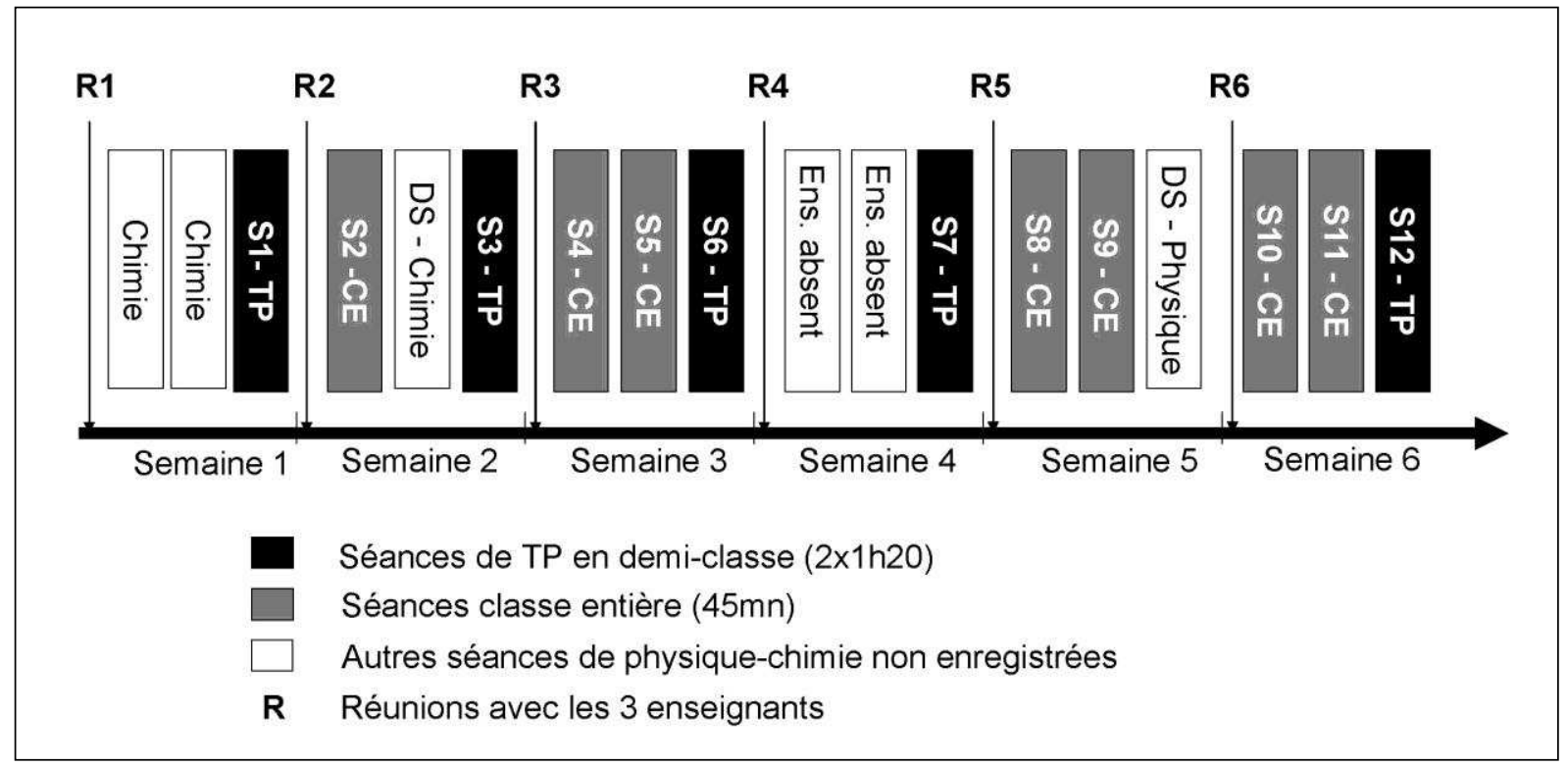




\section{Méthodologie d'analyse}

\section{1) Les réunions entre enseignants}

Elles ont été transcrites en utilisant les conventions développées dans le cadre d'ICOR au sein du laboratoire $\mathrm{ICAR}^{2}$. Nous avons utilisé un tableau à cinq entrées : le temps; le numéro de tour de parole ; le locuteur ; la transcription des verbalisations ; la description des opérations gestuelles avec les ressources.

Notre objectif était ensuite de procéder à une analyse des actions à l'aide d'une grille d'analyse. Pour parvenir à cela, nous avons tout d'abord cherché à regrouper les échanges en fonction de thèmes de discussion, i.e. nous avons effectué des regroupements de plusieurs échanges présentant un fort degré de cohérence sémantique. Trois ensembles de thèmes ont émergé, relativement cohérents avec ce qu'ont pu mettre en évidence d'autres travaux à propos de ce qui préoccupe les enseignants lors des phases préparatoires de cours (Shavelson \& Stern, 1981) : le premier sur l'enseignement, notamment sur le contenu à enseigner et les conditions de mise en œuvre de ces contenus ; le second sur les élèves, leurs difficultés et réactions, leur motivation et leurs connaissances par rapport à ce qui serait proposé ; le troisième, sur sa propre pratique ou celle des autres en matière d'enseignement et de prise en compte des élèves. Puis, en affinant, nous avons peu à peu construit les différents objets de préoccupation des enseignants, sous-jacents à ces thèmes. Nous avons adjoint deux autres propriétés à cette première caractérisation des actions, : leur nature cognitive (analyser ; comparer ; anticiper ; construire) et les ressources mobilisées. Le tableau ci-dessous donne le détail de la grille d'analyse (Figure 3).

Figure 3 : types de préoccupation

\begin{tabular}{|c|c|c|}
\hline Types d'objets de préoccupation & Nature cognitive & Ressources \\
\hline Enseignement : savoir en jeu (à enseigner ou enseigné) & \multirow{8}{*}{$\begin{array}{l}\text { Analyser } \\
\text { Comparer } \\
\text { Anticiper } \\
\text { Construire }\end{array}$} & \multirow{8}{*}{$\begin{array}{l}\text { Internes : connaissances } \\
\text { personnelles des enseignants } \\
\text { Externes : } \\
\text { - documents de présentation de } \\
\text { la séquence et des outils associés } \\
\text { - manuel scolaire } \\
\text { - document préparatoire des } \\
\text { enseignants }\end{array}$} \\
\hline Enseignement : organisation de la classe & & \\
\hline Enseignement : temps-durée de mise en oeuvre & & \\
\hline Élèves : difficultés & & \\
\hline Élèves : réactions & & \\
\hline Élèves : motivation & & \\
\hline Élèves : connaissances & & \\
\hline $\begin{array}{l}\text { Enseignant(s) : pratique(s) d'enseignement et de prise en } \\
\text { compte des élèves }\end{array}$ & & \\
\hline
\end{tabular}

Précisons ici que les enseignants peuvent faire des liens entre différents objets de préoccupation. Par exemple, ils peuvent analyser le savoir en jeu en lien avec les difficultés (prévisibles ou effectivement constatées en cours) des élèves; ou encore, comparer leur pratique par rapport au savoir enseigné.

\section{2) L'activité en classe de l'enseignant A}

Nous avons découpé les séances de classe à l'aide d'un tableau synoptique (cf. figure 4) comprenant plusieurs colonnes : temps (découpage toutes les $2 \mathrm{mn}$ ); mode d'organisation de la classe (classe entière, petits groupes, mixte); activité d'enseignement concernée dans la séquence proposée; type d'action de l'enseignant; ressources mobilisées; description résumée du contenu de chaque action avec le cas échéant une photo de la vidéo.

Figure 4: extrait d'un tableau synoptique d'une séance d'enseignement 


\begin{tabular}{|c|c|c|c|c|c|}
\hline Tps & Org & Activité & Action & Ressources & Description du contenu \\
\hline $0-2$ & $\begin{array}{l}\text { Classe } \\
\text { entière }\end{array}$ & $\begin{array}{l}\text { Activité } 1 \text { de } \\
\text { la partie } 3 \\
\text { «Utilisation }\end{array}$ & $\begin{array}{l}\text { Corre } \\
\text { ction }\end{array}$ & \begin{tabular}{ll|l} 
Discours & de \\
l'enseignant & \\
li & \\
$d$
\end{tabular} & $\begin{array}{l}\text { Le discours de l'enseignant situe le contenu de } \\
\text { la prochaine tâche : correction de l'activité n } 1 \\
\text { de la Partie } 3 \text { de l'enseignement }\end{array}$ \\
\hline $2-4$ & & mécanique » & & $\begin{array}{l}\text { Une partie de laÉ } \\
\text { solution écrite par } \\
\text { un élève est au } \\
\text { tableau }\end{array}$ & Écrit de l'élève : 1 diagramme \\
\hline
\end{tabular}

Nous avons utilisé une typologie des actions du professeur relativement simple, correspondant à 3 étapes de réalisation d'une activité telle que définie dans Sésames :

- Présentation : l'enseignant présente l'activité que lui-même compte faire ou qu'il compte faire faire à ses élèves (par exemple : « aujourd'hui nous allons faire ...).

- Résolution : il répond à la consigne de l'activité ou demande aux élèves d'y répondre (par exemple : « je vous laisse répondre à la question de l'activité ... »).

- Correction : il corrige ou demande à des élèves de corriger une activité qui a été faite auparavant (par exemple : «bon allez on corrige ...).

Puis nous avons analysé les actions de A dans sa classe :

- d'une part en lien avec ses choix dans son document de préparation personnelle (cf. figure 5) et au regard des caractéristiques des ressources Sésames; nous cherchions ce qui a été effectivement repris/non repris/modifié tant du point de vue de l'organisation de classe proposée, que des choix d'activités et de contenus et ce qui a été effectivement mis en œuvre dans la classe ;

- d'autre part, du point de vue des effets de ces choix et actions sur le contrat didactique effectivement à l'œuvre dans la classe.

Figure 5 : extrait de la préparation de l'enseignant

$$
\text { Interactions et forces }
$$

But: ne plus se contenter de décrire le mouvement mais faire un premier pas vers la recherche des causes éventuelles du mouvement.

\section{LES INTERACTIONS :}

Activité 1: Introduction de la notion d'action

Vous disposez du matériel : support, élastique, morceau de métal. Le morceau de métal est suspendu à un fil élastique. Il est immobile.

Questions:

a) Quels sont les objets qui agissent sur le morceau de métal?

b) Sur quels objets agit le morceau de métal ?

rem : préciser la notion d'objet (Pourquoi cette sous-partie) au sens d'objet d'étude : un homme est un objet d'étude donc c'est une notion très large !

- l'élastique agit sur le morceau de métal, la terre aussi ! ( pourquoi le morceau de métal tire-t-il sur l'élastique : volonté personnelle? non! car la Terre l'attire !)

Légende : en italique, signifie une reprise d'un extrait de la partie élève du document OUTILS/PEGASE ; en gras, une reprise de la partie prof de ce même document ; en souligné : quelque chose qui peut être mis en lien avec un échange lors des réunions de préparation avec ses autres collègue.

\section{Présentation des résultats}

Nos résultats sont présentés en trois parties. Tout d'abord nous donnons une vue d'ensemble de l'analyse des réunions. Ensuite nous illustrons les comportements majoritaires observés à partir d'extraits de discussions tirés de ces deux réunions. Dans une troisième partie, plus 
succincte, nous présentons quelques caractéristiques de l'activité de l'enseignant A observée dans sa classe en lien avec l'utilisation des ressources Sésames.

\section{Vue d'ensemble des réunions}

L'analyse systématique des réunions a été faite à partir des caractéristiques des actions présentées dans le cadre théorique. Les données (Figure 6) proposent une vue globale des préoccupations et natures des actions des trois enseignants lors de cinq réunions de groupe destinées à préparer quatre semaines d'enseignement.

Figure 6 : préoccupations initiées par chacun des enseignants au cours des 5 premières réunions. (L'enseignant $A$ était absent à la réunion $R 4$ et $B$ lors des réunions $R 3$ et $R 5$ ).

\begin{tabular}{|l|l|l|l|l|l|l|l|l|l|l|l|l|l|}
\hline & & \multicolumn{3}{|c|}{ A } & \multicolumn{6}{c|}{ C } & \multicolumn{3}{c|}{ B } \\
\hline Nature & Objet(s) & $\mathbf{R 1}$ & $\mathbf{R 2}$ & $\mathbf{R 3}$ & $\mathbf{R 5}$ & $\mathbf{R 1}$ & $\mathbf{R 2}$ & $\mathbf{R 3}$ & $\mathbf{R 4}$ & $\mathbf{R 5}$ & $\mathbf{R 1}$ & $\mathbf{R 2}$ & $\mathbf{R 4}$ \\
\hline Analyse & Savoir & 5 & 5 & 19 & 17 & 4 & 3 & 3 & 11 & 10 & 8 & 7 & 11 \\
\hline & Organisation & & & 1 & 1 & & & & & 1 & & & \\
\hline & Pratique & 2 & 4 & 17 & 15 & 1 & 5 & 7 & 15 & 3 & 5 & 11 & 24 \\
\hline Total & & $\mathbf{7}$ & $\mathbf{9}$ & $\mathbf{3 7}$ & $\mathbf{3 3}$ & $\mathbf{5}$ & $\mathbf{8}$ & $\mathbf{1 0}$ & $\mathbf{2 6}$ & $\mathbf{1 4}$ & $\mathbf{1 3}$ & $\mathbf{1 8}$ & $\mathbf{3 5}$ \\
\hline Compare & Pratique & $\mathbf{5}$ & $\mathbf{1}$ & $\mathbf{0}$ & $\mathbf{0}$ & $\mathbf{3}$ & $\mathbf{1}$ & $\mathbf{0}$ & $\mathbf{0}$ & $\mathbf{0}$ & $\mathbf{2}$ & $\mathbf{1}$ & $\mathbf{0}$ \\
\hline Anticipe & Savoir & 1 & 5 & 3 & 2 & 1 & 3 & & 4 & 3 & 2 & & 5 \\
\hline & Organisation & 2 & 2 & 2 & 1 & 1 & 1 & & & 8 & & & \\
\hline & Savoir et organisation & & 2 & 1 & 1 & & & & 1 & 2 & & 1 & 2 \\
\hline & Difficultés des élèves & & 1 & & & & & & & & & & \\
\hline & Réactions des élèves & & & & 2 & 1 & & & & & 1 & & \\
\hline Total & & $\mathbf{3}$ & $\mathbf{1 0}$ & $\mathbf{6}$ & $\mathbf{6}$ & $\mathbf{3}$ & $\mathbf{4}$ & $\mathbf{0}$ & $\mathbf{5}$ & $\mathbf{1 3}$ & $\mathbf{3}$ & $\mathbf{1}$ & $\mathbf{7}$ \\
\hline Construit & Savoir & $\mathbf{2}$ & $\mathbf{1}$ & $\mathbf{1}$ & $\mathbf{0}$ & $\mathbf{1}$ & $\mathbf{1}$ & $\mathbf{0}$ & $\mathbf{7}$ & $\mathbf{3}$ & $\mathbf{2}$ & $\mathbf{1}$ & $\mathbf{7}$ \\
\hline \multicolumn{2}{|c|}{ Totalité des préoccupations } & $\mathbf{1 7}$ & $\mathbf{2 1}$ & $\mathbf{4 4}$ & $\mathbf{3 9}$ & $\mathbf{1 2}$ & $\mathbf{1 4}$ & $\mathbf{1 0}$ & $\mathbf{3 8}$ & $\mathbf{3 0}$ & $\mathbf{2 0}$ & $\mathbf{2 1}$ & $\mathbf{4 9}$ \\
\hline
\end{tabular}

À la lecture de ce tableau, nous observons que pour chaque enseignant, le nombre total de préoccupations augmente assez nettement entre les deux premières réunions et les suivantes . Le savoir et la pratique constituent des objets de préoccupation dominants. Le tableau suivant (Figure 7) complète cette vue d'ensemble en montrant le pourcentage en nature des actions des trois enseignants et leur évolution au cours des réunions. Ce second tableau montre que toutes les natures d'actions sont présentes et assez réparties lors des deux premières réunions pour les deux enseignants. En revanche à partir de la $3^{\text {ème }}$ réunion, l'analyse devient majoritaire et l'on a vu, dans le tableau précédent (figure 6) qu'elle portait sur le savoir et la pratique des enseignants.

Figure 7 : répartition des différentes natures d'actions de chaque enseignant au cours des 5 premières réunions

\begin{tabular}{|l|l|l|l|l|l|l|l|l|l|l|l|l|}
\hline & \multicolumn{4}{|c|}{ A } & \multicolumn{1}{c|}{ C } & \multicolumn{3}{c|}{ B } \\
\hline \multicolumn{1}{|c|}{ Nature } & \multicolumn{1}{|c|}{ R1 } & \multicolumn{1}{|c|}{ R2 } & R3 & R5 & R1 & R2 & R3 & R4 & R5 & R1 & R2 & R4 \\
\hline Analyse & $41 \%$ & $43 \%$ & $\mathbf{8 4 \%}$ & $\mathbf{8 5 \%}$ & $42 \%$ & $57 \%$ & $\mathbf{1 0 0 \%}$ & $\mathbf{6 8 \%}$ & $\mathbf{4 7 \%}$ & $65 \%$ & $\mathbf{8 6 \%}$ & $\mathbf{7 1 \%}$ \\
\hline Compare & $29 \%$ & $5 \%$ & $0 \%$ & $0 \%$ & $25 \%$ & $7 \%$ & $0 \%$ & $0 \%$ & $0 \%$ & $10 \%$ & $5 \%$ & $0 \%$ \\
\hline Anticipe & $18 \%$ & $48 \%$ & $14 \%$ & $15 \%$ & $25 \%$ & $29 \%$ & $0 \%$ & $13 \%$ & $43 \%$ & $15 \%$ & $5 \%$ & $14 \%$ \\
\hline Construit & $12 \%$ & $5 \%$ & $2 \%$ & $0 \%$ & $8 \%$ & $7 \%$ & $0 \%$ & $18 \%$ & $10 \%$ & $10 \%$ & $5 \%$ & $14 \%$ \\
\hline $\mathrm{N}(100 \%)$ & 17 & 21 & 44 & 39 & 12 & 14 & 10 & 38 & 30 & 17 & 21 & 44 \\
\hline
\end{tabular}

Le tableau suivant (Figure 8) est centré sur le seul enseignant A. Il montre l'importance respective de ses différents objets de préoccupations, le plus souvent combinés dans ses actions (ex : analyse du savoir en lien avec les difficultés des élèves). On s'aperçoit ici que cet 
enseignant n'est pas très différent des deux autres : le savoir pour lui aussi est un objet de préoccupation prépondérant, sur lequel portent les nombreuses analyses réalisées.

Figure 8: objets et nature des préoccupations de A au cours des réunions $R 1, R 2, R 3, R 5$

\begin{tabular}{|l|l|l|l|l|l|l|}
\hline \multicolumn{1}{|c|}{ Objet(s) et spécificités } & Nature & $\mathbf{R} 1$ & $\mathbf{R 2}$ & $\mathbf{R 3}$ & $\mathbf{R 5}$ & Total \\
\hline $\begin{array}{l}\text { Savoirs (à enseigner et enseigné) pour eux-mêmes dans leur } \\
\text { relation }\end{array}$ & Analyse & 2 & 3 & 15 & 14 & $\mathbf{3 4}$ \\
\hline & Anticipe & 3 & 2 & 2 & 7 \\
\hline $\begin{array}{l}\text { Savoir en lien avec les difficultés / connaissances / réactions / } \\
\text { motivations des élèves }\end{array}$ & analyse & 5 & 3 & 3 & 2 & $\mathbf{1 3}$ \\
\cline { 2 - 6 } & anticipe & 1 & 2 & & & $\mathbf{3}$ \\
\hline Savoir en lien avec sa propre pratique d'enseignement & analyse & 2 & 3 & 16 & 15 & $\mathbf{3 6}$ \\
\hline Savoir en lien avec le temps de mise en ouvre & anticipe & & 1 & & $\mathbf{1}$ \\
\hline Temps de mis en œuvre du savoir en lien avec sa propre pratique & analyse & 1 & 1 & & $\mathbf{2}$ \\
\hline Savoir en lien avec l'organisation de la classe & analyse & & 2 & 1 & $\mathbf{3}$ \\
\hline Total des objets relatifs au savoir & & & & $\mathbf{9 9}$ \\
\hline Organisation de l'enseignement d'un savoir & analyse & & 1 & 1 & $\mathbf{2}$ \\
\hline & anticipe & $\mathbf{2}$ & $\mathbf{4}$ & $\mathbf{3}$ & $\mathbf{2}$ & $\mathbf{1 1}$ \\
\hline Total des objets relatifs à l'organisation & & & $\mathbf{1 3}$ \\
\hline Difficultés/réactions/motivation des élèves & anticipe & 1 & & 2 & $\mathbf{3}$ \\
\hline Pratiques par rapport au savoir enseigné, au temps et aux difficultés & compare & $\mathbf{5}$ & $\mathbf{1}$ & $\mathbf{0}$ & $\mathbf{0}$ & $\mathbf{6}$ \\
\hline
\end{tabular}

Cette première approche n'est pas suffisante pour aller très loin dans l'interprétation de ce qui se passe dans ces réunions. C'est pourquoi nous avons également mené un travail plus qualitatif sur chacune des réunions. Nous allons illustrer maintenant des points essentiels de l'activité de préparation collective des enseignants à partir d'extraits tirés de deux réunions : la première et la troisième.

\section{Réunion 1}

Forme de l'enseignement en activités ou en TP-cours

Notons tout d'abord que la position de l'enseignante $\mathrm{C}$ est différente de celles de $\mathrm{A}$ et $\mathrm{B}$ dans la mesure où elle a déjà utilisé les activités de la séquence Sésames l'année précédente. Cette position conduit à ce que les autres lui demandent d'abord d'expliquer comment elle a procédé l'année d'avant. Sa première intervention est claire :

$\mathrm{C}$

J'avais commencé en activité donc en TP euh, j'avais pris des parties de documents, généralement que les activités d'ailleurs et je leur avais fait faire les activités les unes à la suite des autres (0:00:19)

Ainsi sa première préoccupation porte sur la forme de l'enseignement. Elle sera peu reprise lors des réunions ultérieures. C'est le premier point que nous illustrons : il porte plus spécifiquement sur la forme de l'enseignement (cours, TP ou activités) qui est une modification importante des pratiques habituelles d'enseignement suggérée par la séquence.

Un second type d'objet de préoccupation sera repris souvent dans les autres réunions : il s'agit du savoir enseigné et plus particulièrement comment en préciser le sens aux élèves. L'enseignante C précise comment elle a gardé la forme Cours - TP tout en utilisant les activités proposées à la suite de sa première intervention. 
Et en cours j'avais pris ce qu'il y avait dans les documents 1 et 2 j'avais fait une synthèse en cours du modèle dans un style un peu plus formel j'avais dicté je ne les avais pas laissés tout seuls mettre le modèle

A $\quad$ Mais tu ne l'avais pas fait [le cours] entre les activités 1 et l'activité 2 car l'activité 2 parle de trajectoire (0:01:47)

A l'inverse, il est intéressant de noter que A annonce un peu plus loin, qu'il va suivre les propositions du document Sésames :

A Parce que moi j'avais l'intention de commencer en TP aussi et de faire en gros ce que j'avais vu c'est-à-dire le 123 par contre j'aurais utilisé ces docs parce que je les ai alors j'étais vachement emmerdé par ça [activité 5 sur la chronophotographie] qui ne me plait pas du tout (0:05:14)

A noter que le «1, 2, 3 » correspond aux trois premières activités de la partie «Décrire un mouvement avec un modèle ». Cette partie porte sur : (1) Représentation d'un objet par un point, (2) Différentes représentations d'un même objet en mouvement (exemple du constat d'accident) et (3) Caractérisation du mouvement de différents objets. Un texte dit de «modèle » est associé à ces activités. Il donne la définition théorique pour les points concernés et les représentations associées du point qui représente un objet, et de la trajectoire.

Plus loin dans la réunion (0:20:13.8) A précise un peu plus comment il va s’y prendre.

A Je vais plutôt partir sur le sur la première partie après je verrai comment cela se passe distribuer les activités au fur et à mesure qu'il faudra introduire des notions je les introduirai en faisant une partie cours mais je pense que je les enchaînerai je ne ferai pas d'un côté une partie cours d'un côté une partie activité (0:20:28)

Ici A annonce qu'il ne fera pas de distinction entre les séances de cours et de TP mais qu'en revanche, il fera des portions de cours au fur et à mesure. Il ne retient donc pas l'idée de laisser les élèves lire et découvrir par eux-mêmes le «modèle ». Il préfère dicter ces énoncés de savoir. Sur ce dernier point, il s'écarte de la proposition de Sésames, qui considère important que les élèves s'approprient le modèle à partir d'un texte. Par ailleurs, autre point important, il semble que A considère la première séance comme un test : il attend de voir ( «après je verrai ») comment cette façon d'enseigner se passe. Nous présentons plus loin comment A organise son enseignement lors de sa première séance.

\section{Le sens du savoir enseigné}

Cette réunion est aussi l'occasion pour les enseignants de discuter du savoir physique en jeu dans l'enseignement. Leur préoccupation porte non sur la physique pointue de la recherche mais sur des définitions de termes ou des interprétations possibles qui peuvent poser problème aux élèves. Dans l'exemple ci-dessous il s'agit de la différence de sens entre «mouvement » et «trajectoire». La discussion commence par une question de B (0:08:35) et se termine 10 minutes plus tard. L'échange démarre ainsi :

$$
\begin{aligned}
& \text { B Et cela leur semble évident la différence entre mouvement et trajectoire déjà } \\
& \mathrm{C} \quad \text { Ah } \\
& \mathrm{B} \quad \text { Parce qu'ils emploient les deux hein moi je me souviens que ce n'était pas forcément } \\
& \text { évident l'an passé hein }
\end{aligned}
$$

La discussion se poursuit par différentes propositions dont certaines font appel à des savoirs physiques assez élaborés. Les trois enseignants vont alors chercher les définitions des termes 
dans un dictionnaire et cherchent leur étymologie, mais ils ne disposent que du petit Larousse. Vers la fin ils s'entendent pour considérer que la trajectoire représente le mouvement d'un point particulier de l'objet (sans que ce soit très explicite).

\section{Réunion 3}

Comme nous l'avons présenté précédemment cette réunion marque un tournant dans la nature des actions. Celles-ci deviennent beaucoup plus centrées sur l'analyse (Figure 8).

Nous illustrons, par des extraits tirés de la troisième réunion, des actions relatives à l'analyse du savoir enseigné du point de vue de sa mise en œuvre dans la classe. Ce type d'action est fréquent dans ces réunions. Dans la partie suivante, nous illustrons aussi la façon dont les enseignants utilisent les documents Sésames pendant les réunions.

Mise en auvre d'éléments du savoir ou démarche de la physique

Un sujet de discussion abordé au cours de la troisième réunion concerne la mise en œuvre de différents éléments de savoir. Nous présentons d'abord un extrait dans lequel les enseignants discutent d'une question délicate de l'enseignement de la physique : ce que le physicien néglige dans une situation quand il l'interprète. Dans l'extrait ci-dessous, il s'agit de l'action de l'air sur les objets que l'on peut, ou non, négliger selon les situations. Cet extrait montre comment les professeurs réfèrent à leurs propres actions en classe et à celles de leurs élèves. Nous avons indiqué en caractères gras les passages dans lesquels les élèves sont mis en jeu et souligné chaque fois que le professeur s'imagine en train d'enseigner.

(0:02:02) alors insignifiantes ou signifiantes ça ne se heu heu le critère définitif [de négliger ou pas l'action de l'air] c'est il est quantitatif. Tu peux pas vraiment aller jusqu'au bout

C $\quad$ Et puis eux ils peuvent pas le dire tout seuls

B Voilà ils peuvent pas le dire tout seuls

A Moi l'air j'ai dit si j'ouvre la fenêtre y'a un courant d'air, oui effectivement vous allez voir le l'objet bouger heu vous allez voir une manifestation de cette action là

B Et... (soupir) elles existent quand même mais...

A Ouais mais attends là là on les emmène dans le mur là

B Et...

C Ben oui mais y'a des fois tu vas la considérer l'action de l'air et pas d'autres

$[\ldots]$

A $\quad . . . \mathrm{d}$ ans tout le, dans tout le, enfin pour l'instant à chaque fois qu'on a, toutes les démarches qu'il sait, on a quasiment jamais parlé de ces frottements on les néglige tout le temps quoi

$[\ldots]$

C $\quad$ T'as jamais mis l'air (?)

A Non

$[\ldots]$

A Ah non j'en suis pas arrivé encore au médecine-ball, non mais avec eux pour l'instant j'ai pas mis l'air, on va le mettre dans l'histoire du motard qui se déplace à vive allure, par exemple tu vois dans le texte (A regarde ses feuilles et les tourne, ressource : Document papier) on insiste sur le fait que le motard il est pas simplement sur sa moto mais qu'il est à vive allure alors...

C Hum hum

A A mon sens moi j'ai traduit vive allure par je peux pas négliger le vent (0:03:43)

Dans cet extrait les enseignants partagent un point de vue commun : les élèves ne peuvent pas décider eux-mêmes s'il faut ou non négliger l'air. A est d'accord: il a décidé de ne pas introduire l'air et ne le fera que si l'on ne peut pas le négliger. Dans ce dernier cas, même si ce 
n'est pas tout à fait explicite, il considère que les élèves n'auront probablement pas de problème à l'accepter (il dit «on va le mettre » et non je vais le mettre, il inclut les élèves implicitement). Son argument «moi l'air j'ai dit» met en jeu une action qu'il a faite en classe. De plus il englobe souvent dans son action, celles des élèves comme par exemple «avec eux pour l'instant j'ai pas mis l'air».

Un autre exemple montre que les professeurs sont sensibles au fait que les éléments de savoir puissent être proposés par les élèves.

A (0:11:27) Et heu et donc il faut arriver à distinguer la terre du sol parce qu'ils ont pas la même action....

C Huhum

A $\quad$... y'a une qu'attire et l'autre qui r'pousse quoi ...

C Huhum

A ... en gros, ça alors c'est venu d'eux à chaque fois j'crois, ils étaient partis sur la terre sur l'objet enfin sur le sur la terre et l'objet parce que c'était le même heu (0:11:42)

Dans ces différentes discussions, les trois enseignants ne remettent pas en cause les activités de la séquence proposée. Ils discutent de leur mise en œuvre dans la classe et, pour cela, mènent une action d'analyse du savoir mais aussi de ce que les élèves peuvent ou non comprendre, prendre en compte ou proposer pour contribuer à la construction de ce savoir dans la classe. Par exemple on a vu dans l'extrait sur l'action de l'air que C, aussi bien que A, considère que les élèves ne peuvent pas, soit comprendre, soit proposer le rôle de l'air. Pour ces professeurs il s'agit d'une vraie difficulté d'enseignement. On peut dire que leur préoccupation principale porte ici sur la mise en œuvre du savoir enseigné dans la classe.

\section{Utilisation de rubriques des documents Sésames}

Nous donnons trois exemples d'utilisation de rubriques proposées dans les documents Sésames : tout d'abord d'une activité, puis d'un commentaire sur la préparation de la séance et enfin d'un commentaire sur le comportement des élèves.

Ce premier exemple illustre l'utilisation faite dans les réunions des documents élève de Sésames, c'est-à-dire ceux donnant les activités. Il s'agit de l'activité n ${ }^{\circ} 7$ (« mise en œuvre de l'ensemble du modèle des interactions »), à propos d'un dispositif déjà utilisé pour introduire la notion d'action (un fil élastique attaché à un support et une pierre), réalisée après l'introduction de la force

A (en lisant l'activité 7) à l'aide du modèle compléter le tableau suivant diagramme interactions voir activité 2

C Représentation des forces modélisant l'interaction pierre terre

A La pierre agit ah d'accord l'interaction donc OK donc tu dois à la fois représenter la force qu'exerce la pierre sur la terre...

$[\ldots]$

C Ah donc tu tu représentes la pierre pis après toc, moi j'l'avais fait en cours ça

[...]

A ...l'interaction pierre terre, l'interaction élastique pierre et après tu regardes les forces que tu as sur la pierre...

$[\ldots]$

A D'accord effectivement t'en as 2 [forces] et après tu heu...

C $\quad$ Et après tu considères juste le système pierre et tu additionnes les 2

A Hum alors moi ce qui m'embête un peu avec cette démarche là j'sais pas si ça si ça va éclaircir ou pas c'est que on parle beaucoup d'interactions on est en train, là on est en 
train de distinguer entre le principe des interactions et puis ce qui va se cacher derrière le heu un bilan de forces avec le principe d'inertie etc...

Cet extrait montre la façon dont les enseignants s'approprient l'activité. Dans le cas de cette activité, la réalisation consiste à faire des diagrammes et des schémas de force. Les enseignants travaillent sur la correction et la commentent. A la fin de l'échange, A pense à une difficulté possible des élèves qui consisterait à faire une confusion entre d'une part, l'égalité de l'action et la réaction qui portent sur deux systèmes différents (si A agit sur $\mathrm{B}, \mathrm{B}$ agit sur $\mathrm{A} ; \mathrm{F}_{\mathrm{A} / \mathrm{B}}=\mathrm{F}_{\mathrm{B} / \mathrm{A}}$ ) et d'autre part, l'égalité des forces qui s'exercent sur un même système si le système est immobile ou en mouvement rectiligne uniforme. Il s'agit là d'une difficulté recensée dans les travaux en didactique et reprise souvent en formation des maîtres. Cette réaction montre que $\mathrm{A}$, mais (aussi probablement) les deux autres, quand ils explicitent la résolution, ont en tête les réactions possibles de leurs élèves.

Cet extrait illustre qu'à ce stade de l'utilisation des documents Sésames ( $3^{\text {ème }}$ réunion, au moins deux séances d'enseignement réalisées) les enseignants sont préoccupés par la compréhension des éléments proposés dans les documents Sésames plus que par une remise en cause de l'activité. On peut dire que les enseignants ont une certaine confiance dans les documents, même si quelques activités sont modifiées.

Un deuxième exemple intéressant porte sur l'utilisation d'un commentaire sur la préparation d'une activité essentielle de la séquence qui nécessite l'utilisation d'un médecine-ball. Juste avant l'extrait suivant, $C$ explique que l'an dernier, elle n'a pas pris de médecine-ball mais des balles légères pour plusieurs raisons : notamment parce qu'il semble que cet équipement n'existe pas dans l'établissement; et parce que cela fait moins de bruit ( $J^{\prime}$ 'ai des petites balles, bleues, ça fait moins de bruit quand ça tombe »), ce qui pourrait rejoindre une préoccupation de garder un certain calme dans la classe.

A (0:14:51) ben en regardant le doc là [commentaire sur la préparation] ils ont l'air de dire que c'est vachement important que l'élève manipule le truc... [ressource document papier]

$\mathrm{C}$ qu'il manipule

A ... qu'il sente quand il exerce la force et quand il exerce plus (0:14:58)

Par la suite, A propose un avis contraire à $\mathrm{C}$ en insistant sur l'importance d'avoir une balle lourde pour qu'à la réception, le mouvement effectif des mains soit vers le bas et donc en sens inverse de l'action de ces mêmes mains décrite dans le commentaire des documents Sésames. On peut noter que lors de l'enseignement de cette activité, A prend un médecine-ball même s'il n'y en a qu'un dans la classe (il fait cette activité en classe dédoublée).

Le troisième exemple montre que certains commentaires sur le comportement des élèves sont déjà connus des enseignants et ne font que renforcer ce type de connaissance.

A (0:23:31) ouais ouais ouais, ça on récupère tout le temps ça jusqu'à la terminale on a ou la vitesse comme une force, ce matin y'en a un qui m'a fait et c'est un meilleur élève il me fait, monsieur, la vitesse et tout, en terminale

C ouais

A oh il s'est fait jeter quand même par les autres, on t'a déjà dit que c'était pas une force (rires) (0:23:47)

A nouveau cet extrait illustre la confiance que A accorde à ces documents.

\section{L'activité d'enseignement de A}


Nous présentons les aspects les plus marquants de l'analyse de l'activité de A dans sa classe, du point de vue de ce qu'il reprend et s'approprie des ressources Sésames et met en œuvre dans sa classe, ainsi que quelques effets constatés sur l'activité des élèves.

Notre analyse, basée sur le document préparatoire de l'enseignant et ses actions effectives en classe, fait ressortir que l'enseignant A reprend 16 des 19 activités proposées dans le document Sésames. Sur les trois activités non reprises, deux sont dans la première partie :

- l'activité 2, intitulée «Différentes représentations d'un même objet en mouvement », qui met en jeu un constat d'accident ;

- l'activité 4 sur la chronophotographie qu'il avait trouvée trop «mathématique » (voir ci-dessus).

La dernière est un exercice de la partie 2.

En ce qui concerne les modèles, A les a repris dans ses documents personnels au titre près.

En ce qui concerne la forme de l'enseignement proposée, en activités plutôt qu'en TP cours, A a mis en oeuvre les activités de telle sorte que les élèves travaillent à deux et puissent lors de la correction exprimer leurs propositions. Ainsi lors de la première séance, quand il a introduit l'activité 1 ( informations conservées ou perdues sur le mouvement d'une balle si on la représente par un point), il a établi un contrat didactique explicite :

P $\quad(0: 09: 17)$...(en distribuant le texte de l'activité qu'il a introduite au préalable) j'aimerais bien que vous essayiez de le tenir le plus longtemps possible ça c'est sur ces documents que vous y mettiez à deux toujours la même chose j'aimerais que vous mettiez la même chose que votre voisin ou votre voisine ce qui sous entend qu'il va falloir vous mettre d'accord avant de l'écrire dans ces cases là j'aimerais que vous y mettiez la même chose.

Le travail en groupe dure 10 minutes sans que le professeur ne s'adresse à toute la classe ; les élèves travaillent donc en petits groupes de manière autonome. Ainsi dès la première activité le professeur «joue le jeu» d'une activité proposée par Sésames. L'analyse de la vidéo montre que la classe aussi «joue le jeu » : les élèves sont actifs pendant le travail en groupe, cherchent des idées et ensuite lors de la mise en commun participent. Ainsi le professeur prend au début la position de l'animateur du débat et non de celui qui donne le savoir. La correction en classe entière démarre ainsi :

$\mathrm{P} \quad$ (0:16:48) on essaie de reprendre ça ensemble voir ce que vous avez mis sur ces papiers [...] c'est bon pour tout le monde [...] Qu'est ce que qu'est ce que vous avez dit sur l'histoire de la balle de heu de tennis alors quelles sont les informations perdues ou conservées qu'est ce que vous commencez par quoi

$\mathrm{E} \quad$ la rotation sur elle-même

$\mathrm{P} \quad$ rotation sur elle-même et conservée

E la translation (inaudible)

$\mathrm{P} \quad$ ça c'est leur opinion vous n'êtes pas obligés d'avoir les mêmes j'ai pas dit que c'était juste j'ai pas dit que c'était faux.

On peut dire que cette première séance test a été considérée de manière positive et qu'une certaine confiance dans les documents s'est installée.

Cependant, alors qu'il est proposé de distribuer le texte du modèle, le professeur fait un exposé et dicte, ceci aussi bien lors de la première séance que pour les suivantes. Il est ici cohérent avec ce qu'il avait annoncé lors des réunions préparatoires avec ses collègues.

L'analyse systématique à l'aide des trois catégories d'action définies (présentation des activités d'enseignement; résolution; correction), croisée avec celle des modes 
d'organisation (classe entière, petites groupes, mixte) montre que, pour une grande majorité d'activités Sésames, l'enseignant a laissé les élèves travailler en groupe conformément aux recommandations émises dans Sésames. Toutefois, il a pris beaucoup de temps en classe entière pour la mise en commun et la correction. Le temps de travail en groupe reste assez minoritaire par rapport à la configuration classe entière (Figure 9)

Figure 9: mode d'organisation de la classe tout au long de la séance

\begin{tabular}{|l|r|r|}
\hline Durée totale de la séquence : 10h 01 mn 52s & & \\
\hline \multicolumn{1}{|c|}{ Mode d'organisation de la classe } & \multicolumn{1}{c|}{ Durée } & \multicolumn{1}{c|}{$\%$} \\
\hline Classe entière & $7: 39: 28$ & 76,37 \\
\hline Groupe & $2: 02: 44$ & 20,39 \\
\hline Mixte & $0: 19: 31$ & 3,24 \\
\hline
\end{tabular}

En revanche, A ne semble pas s'être approprié un aspect épistémologique essentiel de la conception des séquences Sésames : celui de la modélisation en physique. L'hypothèse liée à cette approche de la modélisation est qu'il est nécessaire de distinguer la description d'une situation matérielle et son interprétation en termes de concepts physiques.

On peut prendre pour exemple de cette difficulté d'appropriation, le moment où A introduit le concept de force (séance 5). Pour les concepteurs de Sésames, la force est un concept physique qui rend compte de l'action d'un objet sur un autre. Il est donc proposé de décrire la situation matérielle en termes d'action puis de la modéliser en termes de forces. Cette distinction est rendue explicite dans la séquence par un texte appelé «modèle » qui introduit la force comme un concept qui permet de représenter l'action. A utilise ce texte dans la classe pour introduire la force, mais, dans sa présentation, apparait une différence entre son discours de commentaire du texte et le texte lui-même . Cet extrait se situe à la fin de la correction d'une activité où il s'agit d'utiliser un diagramme « système interaction » pour représenter les phases de lancer et de réception d'un médecine-ball; les objets sont représentés par des « patates », les interactions de contact entre objets par des flèches à deux sens pleines et les interactions à distance par des flèches à deux sens en pointillés. . Les diagrammes représentant ces deux phases sont les mêmes. L'argument est donc qu'il faut prendre un modèle plus élaboré pour rendre compte de la différence. physique les simples interactions ça ne va pas suffire [...] mais il va falloir passer à une étape suivante pour essayer de traduire ces interactions avec un autre heu outil cet outil ben il était énoncé dans le titre dès le départ qu'est ce que j'avais mis comme titre $(0: 31: 13) j^{\prime}$ 'avais mis quoi comme titre à interaction j'avais mis force [...] et puis là on va parler de ce qu'est heu une force vous avez croisé ça en

E jamais

$\mathrm{P} \quad$ en 3ème ( ?)

E (inaudible)

$\mathrm{P} \quad$ jamais ça ne dit rien à personne ça donc je vais vous donner la suite du modèle on va lire ensemble et puis on va voir comment on peut utiliser ça (P prend un tas de feuilles et commence à le distribuer) Pour l'instant on a parlé d'interactions dans le modèle (en distribuant)

$\mathrm{P} \quad$ alors on lit ensemble il faut bien l'intégrer ce modèle allez lisez bien ça en application vous allez voir que ça n'a rien de compliqué ça peut paraître un petit peu plus ardu à la lecture force ( $P$ lit) quand un système $X$ est en interaction avec un système A on appelle force exercée par A sur X l'action de A sur $X$ vous voyez la bascule qu'on est en train de faire le terme d'action finalement on est en train de le remplacer par le terme de force d'accord l'intérêt c'est que l'action on la représentait d'une manière encore assez vague et la force on va avoir un outil mathématique très précis pour la représenter (0:33:11) 
Dans cet extrait, il apparaît que la formulation des commentaires du professeur A exprime l'idée qu'on remplace l'action par la force (en gras dans la dernière intervention), ce qui est en désaccord avec l'idée d'introduction d'un modèle plus pertinent, justifiée par les limites du précédent. Dans ce cas, le diagramme «système interaction » est un modèle insuffisamment élaboré et il faut prendre un autre modèle. Ainsi dans le discours du professeur A il y a coexistence de son approche (remplacement de l'action par la force) et de celle des concepteurs de la séquence Sésames par l'intermédiaire du texte que le professeurA a repris intégralement (qui pose que $\mathrm{F}$ est une représentation de l'action et non plus de l'interaction).

D'ailleurs, le professeur A, qui a poursuivi après cette étude l'utilisation de ces séquences et a participé de plus en plus activement au groupe Sésames a reconnu qu'il n'avait pas vu et pris en compte cet aspect épistémologique cette année là.

\section{Conclusion}

De cette étude fine et longitudinale, centrée sur trois enseignants lors de la phase de préparation, et sur l'activité de l'un d'entre eux dans sa classe, il ressort des points importants relatifs à la réception puis la mise en œuvre, par des enseignants, d'une nouvelle séquence d'enseignement.

La question de la faisabilité de la mise en œuvre de la séquence proposée est une préoccupation première: lors de la première réunion, les tous premiers échanges sont consacrés à l'explication, par l'enseignante $\mathrm{C}$, de la manière dont elle a mis en œuvre la séquence l'année précédente. Elle le fait en réponse au questionnement de ses deux collègues, ce qui montre qu'il s'agit pour eux d'une préoccupation majeure. Mais pour l'enseignant A, cela n'est pas suffisant: il considère très explicitement que les toutes premières séances (première et deuxième séances) constituent un moment de test très important pour juger de la faisabilité concrète dans sa classe. Une fois cette phase de test passée et réussie (les élèves jouent le jeu proposé et «ça passe » du point de vue des contraintes temporelles), les préoccupations de l'enseignant $\mathrm{A}$ et de ses collègues changent pour se concentrer sur la mise en œuvre du savoir. Il ne s'agit plus de questionner les activités et le mode d'organisation proposés du point de vue de leur faisabilité, mais de s'interroger sur les potentielles difficultés des élèves quant à des éléments de savoir et de trouver les moyens de dépasser celles-ci. Nous pouvons donc faire l'hypothèse que la confiance dans la ressource proposée doit pouvoir se créer très rapidement chez l'enseignant (dès la première séance), étant donné le poids des contraintes qui pèsent sur la classe (matériel disponible, impact du mode d'organisation de la classe proposé sur l'engagement des élèves et l'ordre dans la classe ; progression vis-à-vis du programme) et qui ne permettent pas de rester longtemps en phase expérimentale. De ce point de vue, la ressource proposée semble efficace : cette étude conforte d'ailleurs les retours fournis par plusieurs autres enseignants participant aux groupes de Recherche \& Développement Sésames.

L'étude montre également que les ressources proposées ne sont pas reprises en bloc mais que les enseignants cherchent à les articuler à leur propre activité en conservant des aspects de celles-ci qui leur semblent probablement importants : en particulier, on a vu comment $\mathrm{A}$ gardait des moments pour dicter des éléments de savoir au lieu de donner le texte écrit du modèle aux élèves afin qu'ils se l'approprient au cours de présentations, activités, exercices, discussions et débats. On peut aussi considérer que le processus d'appropriation par les enseignants de la séquence va prendre du temps et que ce qui prime, dans un premier temps, c'est la mise en œuvre de cette dernière, même de manière incomplète, sans toutefois perdre ce qui fait le cœur de ces ressources. Nos analyses montrent d'ailleurs que, même si elle n'est 
pas mise en œuvre dans sa totalité, la séquence produit déjà des effets intéressants chez les élèves sur le plan de l'engagement dans la situation proposée.

Une difficulté majeure apparaît cependant dans cette première phase du processus d'appropriation pour A : lui, comme ses deux collègues, n'ont saisi que très partiellement l'approche épistémologique spécifique de la séquence proposée basée sur une certaine conception de la modélisation en physique (Tiberghien, et al., 2009). On touche sans doute là la limite la plus importante d'une appropriation sur la seule base de documents écrits. L'expérience des groupes de Recherche \& développement Sésames, intégrant des enseignants, montre bien que cette compréhension fine des soubassements épistémologiques de l'approche proposée peut prendre plusieurs années et nécessite de nombreux échanges entre chercheurs et enseignants. L'enseignant A lui-même, s'est engagé dans ces groupes et a largement évolué par la suite.

\section{Bibliographie}

ALTET, M. (1994). La formation professionnelle des enseignants. Paris: P.U.F.

ALTET, M., \& VINATIER, I. (2008). Analyser et comprendre la pratique enseignante. Rennes : Presses Universitaires de Rennes.

AMIGUES, R. (2002). L'enseignement comme travail. In P. Bressoux (Ed.), Note de Synthèse pour Cognitique : Programme école et Sciences Cognitives, pp243-262.

AMIGUES, R., \& FAÏTA, D. (2001). La question du collectif dans le travail des professeurs. Paper presented at the 4ème congrès international, actualité de la recherche en éducation, Lille.

BRESSOUX, P., AMIGUES, R., ARNOUX, M., BARRE DE MINIAC, C., CLANET, J., DESSUS, P. (2002). Les stratégies de l'enseignant en situation d'interaction. In P. Bressoux (Ed.), Note de Synthèse pour Cognitive, Programme école et sciences cognitives. Ministère de la Recherche.

BRONFENBRENNER, U. (1986). Dix années de recherches sur l'écologie du développement humain. In M. Crahay \& D. Lafontaine (Eds), L'art et la science de l'enseignement. Bruxelles : Labor, pp283-301.

BROPHY, J. E. (1983). Research on the self-fulfilling prophecy and teacher expectations. . Journal of Educational Psychology, Vol. 5, No 5, pp631-661.

BROUSSEAU, G. (1998). Théorie des situations didactiques. Grenoble: La pensée sauvage.

BRU, M. (1991). Les variations didactiques dans l'organisation des conditions d'apprentissage. Toulouse : Editions Universitaires du Sud.

CLOT, Y. (1999). La fonction psychologique du travail. Paris : PUF.

COULAUD, M. (2005). Evaluer la compréhension des concepts de mécanique chez des élèves de secondes: développement d'outils pour les enseignants. Thèse de doctorat, Université de Lyon : Université Lyon 2.

CRAHAY, M. (1989). Contraintes de situation et interactions maître-élève, changer sa façon d'enseigner, est-ce possible ? Revue Française de Pédagogie No 88, pp67-94.

DOYLE, W. (1983). Academic work. Review of Educational Research, Vol. 53, No 2, pp159199.

DOYLE, W. (1986). Classroom organization and management. In W. M.C. (Ed.), Handbook of research on teaching New York: McMillan, pp392-431.

DURAND, M. (1996). L'enseignement en milieu scolaire. Paris : PUF.

GAL-PETITFAUX, N., \& SAURY, J. (2002). Analyse de l'agir professionnel en EPS dans une perspective d'anthropologie cognitive. Revue Française de Pédagogie, No 138, pp5161. 
GUEUDET, G., \& TROUCHE, L. (2009). Vers de nouveaux systèmes documentaires des professeurs de mathématiques. In I. Bloch \& F. Conne (Eds), Nouvelles perspectives en didactique des mathématiques. Grenoble : La Pensée Sauvage, pp109-133.

GUEUDET, G., \& TROUCHE, L. (2010). Ressources vives, le travail documentaire des professeurs en mathématiques. Rennes : Presses Universitaires de Rennes.

JEANNIN, L. (2006). Appropriation par un enseignant de physique d'une nouvelle séquence d'enseignement: cas de la mécanique en seconde. Thèse de doctorat, Université de Lyon : Université Lyon 2.

KÜÇÜKÖZER, A. (2005). L'étude de l'évolution de la compréhension conceptuelle des élèves avec un enseignement. Cas de la mécanique en lère S. Thèse de doctorat, Université de Lyon : Université Lyon 2.

LAGRANGE J.B., LECAS J.F., \& PARZYSZ B (2006 ). Les professeurs stagiaires d'IUFM et les technologies. Quelle instrumentation? Recherche et Formation, No 52, pp131-147.

LAHIRE, B. (1998). L'homme pluriel. Paris : Nathan.

LEINHARDT, G., \& GREENO, T. (1986). The cognitive skills of teaching. Journal of Educational psychology, Vol. 78, No 2, pp75-95.

LEONTIEV, A. N. (1979). The problem of activity in psychology. In J. V. Wertsch (Ed.), The concept of activity in soviet psychology. New York : M.E. Sharpe.

ROBERT, A. (1999). Recherches didactiques sur la formation professionnelle des enseignants de mathématiques du second degré et leurs pratiques en classe. Didaskalia, No 15, pp123-157.

ROBERT, A., \& ROGALSKI, J. (2005). A cross-analysis of the mathematics teachers' activity. An example in a French 10th-grade class. Educational Studies in Mathematics, Vol. 59, No 1, pp269-298.

ROGALSKI, J. (2000). Y'a-t-il un pilote dans la classe ? Apports des concepts et des méthodes de la psychologie ergonomique pour l'analyse de l'activité enseignante. Paper presented at the 26ème colloque COPIRELEM, Limoges.

SHAVELSON, R. J., \& STERN, P. (1981). Research on teachers pedagogical thought, judgments, decisions and behavior. Review of Educational Research, Vol. 51, No 4, pp455-498.

STIGLER, J., W., GONZALES, P., KAWANAKA, T., KNOLL, S., \& SERRANO, A. (1999). The TIMSS Videotape Classroom Study: Methods and Findings from an Exploratory Research Project on Eighth-Grade Mathematics Instruction in Germany, Japan, and the United States. Washington DC: National Center for Education Statistics Office of Educational Research and Improvement U.S. Department of Education.

TIBERGHIEN, A., VINCE, J., \& GAIDIOZ, P. (2009). Design-based research: case of a teaching sequence on mechanics. International Journal of Science Education, Vol. 31, No 17, pp2275-2314.

TOCHON, F. (1993). L'enseignant expert. Paris : Nathan.

WALBERG, H. J., \& FOWLER, W. J. (1991). School size, characteristics, and outcomes. Educational Evaluation and Policy Analysis. Vol. 13, No 2, pp189-202. 